\title{
Quality, Features, and Presence of Behavior Change Techniques in Mobile Apps Designed to Improve Physical Activity in Pregnant Women: Systematic Search and Content Analysis
}

Melanie Hayman ${ }^{1}$, PhD; Kristie-Lee Alfrey ${ }^{1}$, BPsycSc(Hons); Summer Cannon ${ }^{1}$, BPsycSc(Hons); Stephanie Alley ${ }^{1}$, PhD; Amanda L Rebar ${ }^{1}, \mathrm{PhD}$; Susan Williams ${ }^{1}$, PhD; Camille E Short ${ }^{2}$, PhD; Abby Altazan ${ }^{3}, \mathrm{MSc}$; Natalie Comardelle ${ }^{3}$, BSc; Sinead Currie ${ }^{4}$ PhD; Caitlin Denton ${ }^{3}$, BSc; Cheryce L Harrison ${ }^{5}, \mathrm{PhD}$; Tayla Lamerton ${ }^{6}$ PhD; Gabriela P Mena ${ }^{6}$, MD; Lisa Moran ${ }^{5}, \mathrm{PhD}$; Michelle Mottola ${ }^{7}, \mathrm{PhD}$; Taniya S Nagpal ${ }^{8}$; Lisa Vincze ${ }^{9}, \mathrm{PhD}$; Stephanie Schoeppe ${ }^{1}, \mathrm{PhD}$

\footnotetext{
${ }^{1}$ School of Health, Medical and Applied Sciences, CQUniversity, Rockhampton, Australia

${ }^{2}$ School of Psychological Sciences, University of Melbourne, Melbourne, Australia

${ }^{3}$ Pennington Biomedical Research Center, Louisiana State University, Baton Rouge, LA, United States

${ }^{4}$ Division of Psychology, Stirling University, Scotland, United Kingdom

${ }^{5}$ School of Public Health and Preventive Medicine, Monash University, Melbourne, Australia

${ }^{6}$ School of Human Movement and Nutrition Sciences, University of Queensland, Brisbane, Australia

${ }^{7}$ Faculty of Health Sciences in Kinesiology, University of Western Ontario, London, ON, Canada

${ }^{8}$ School of Human Kinetics, University of Ottawa, Ottawa, ON, Canada

${ }^{9}$ School of Allied Health Sciences - Nutrition and Dietetics, Griffith University, Gold Coast, Australia
}

\section{Corresponding Author:}

Melanie Hayman, PhD

School of Health, Medical and Applied Sciences

CQUniversity

Bruce Highway

Rockhampton, 4701

Australia

Phone: 6149306912 ext 56912

Email: m.j.hayman@cqu.edu.au

\section{Abstract}

Background: Physical activity during pregnancy is associated with several health benefits for the mother and child. However, very few women participate in regular physical activity during pregnancy. eHealth platforms (internet and mobile apps) have become an important information source for pregnant women. Although the use of pregnancy-related apps has significantly increased among pregnant women, very little is known about their theoretical underpinnings, including their utilization of behavior change techniques (BCTs). This is despite research suggesting that inclusion of BCTs in eHealth interventions are important for promoting healthy behaviors, including physical activity.

Objective: The aim of this study was to conduct a systematic search and content analysis of app quality, features, and the presence of BCTs in apps designed to promote physical activity among pregnant women.

Methods: A systematic search in the Australian App Store and Google Play store using search terms relating to exercise and pregnancy was performed. App quality and features were assessed using the 19-item Mobile App Rating Scale (MARS), and a taxonomy of BCTs was used to determine the presence of BCTs (26 items). BCTs previously demonstrating efficacy in behavior changes during pregnancy were also identified from a literature review. Spearman correlations were used to investigate the relationships between app quality, app features, and number of BCTs identified.

Results: Nineteen exercise apps were deemed eligible for this review and they were accessed via Google Play (n=13) or App Store $(n=6)$. The MARS overall quality scores indicated moderate app quality (mean 3.5 [SD 0.52]). Functionality was the highest scoring MARS domain (mean 4.2 [SD 0.5]), followed by aesthetics (mean 3.7 [SD 0.6]) and information quality (mean 3.16 [SD 0.42]). Subjective app quality (mean 2.54 [SD 0.64]) and likelihood for behavioral impact (mean 2.5 [SD 0.6]) were the lowest scoring MARS domains. All 19 apps were found to incorporate at least two BCTs (mean 4.74, SD 2.51; range 2-10). However, only 11 apps included BCTs that previously demonstrated efficacy for behavior change during pregnancy, the most common 
being provide opportunities for social comparison $(\mathrm{n}=8)$ and prompt self-monitoring of behavior $(\mathrm{n}=7)$. There was a significant positive correlation between the number of BCTs with engagement and aesthetics scores, but the number of BCTs was not significantly correlated with functionality, information quality, total MARS quality, or subjective quality.

Conclusions: Our findings showed that apps designed to promote physical activity among pregnant women were functional and aesthetically pleasing, with overall moderate quality. However, the incorporation of BCTs was low, with limited prevalence of BCTs previously demonstrating efficacy in behavior change during pregnancy. Future app development should identify and adopt factors that enhance and encourage user engagement, including the use of BCTs, especially those that have demonstrated efficacy for promoting physical activity behavior change among pregnant women.

(JMIR Mhealth Uhealth 2021;9(4):e23649) doi: $\underline{10.2196 / 23649}$

\section{KEYWORDS}

pregnancy; exercise; physical activity; mobile health (mHealth); applications; MARS; behavior change techniques; mobile phone

\section{Introduction}

Physical activity during pregnancy is associated with a variety of health benefits, including reduced risk of excessive gestational weight gain, gestational diabetes, gestational hypertension, preeclampsia, the severity of pelvic girdle pain, macrosomia, instrumental delivery, postpartum weight retention, urinary incontinence, and depressive disorders [1,2]. Despite the many health benefits of physical activity during pregnancy, few women participate in regular physical activity during pregnancy [3]. In addition, women tend to reduce or cease their participation in physical activity once they become pregnant and throughout the course of their pregnancy [3,4]. This may be as a result of various barriers, including mother-child safety concerns, fatigue, change in body shape, and associated pain. Further, a lack of provision of adequate information, knowledge, social support, and self-efficacy for behavior change are other issues that may exacerbate the decline in activity levels throughout pregnancy $[2,5]$.

There are many avenues for women to access information and support related to maintaining a healthy pregnancy, including information about physical activity behaviors. Historically, pregnant women have accessed information from doctors, midwives, family, and friends to guide and inform their physical activity behaviors. However, eHealth platforms such as the internet and mobile apps are now altering the way women access this information [6] and they have become an important information source for pregnant women $[7,8]$. In fact, a recent Australian study among 410 pregnant women investigated the use of pregnancy and parenting apps and found that almost three-quarters of the studied women used at least one of these types of pregnancy apps [9]. In addition, more than half of the participants reported using 2-4 apps throughout their pregnancy. The frequency of app use was also significant, with almost a quarter of pregnant women reporting daily use of apps [9]. While the use of pregnancy-related apps has significantly increased among pregnant women [8], very little is known about their theoretical underpinnings, including their utilization of behavior change techniques (BCTs). This is despite research suggesting that inclusion of BCTs in eHealth interventions can play an important role in improving, supporting, and maintaining healthy behaviors, including physical activity $[10,11]$.

In 2013, Currie et al [12] systematically evaluated the content of physical activity interventions designed to reduce the decline of physical activity in pregnant women with a specific emphasis on BCTs [13] employed to elicit this change. Six common BCTs shown to have some efficacy in improving physical activity behaviors were identified: prompt intention formation, prompt specific goal setting, prompt review of behavioral goals, prompt self-monitoring of behavior, provide feedback on performance, and provide opportunities for social comparison [12]. Since this review, many behavior change interventions have used these BCTs to promote positive physical activity behaviors among pregnant women [14].

Previous reviews of physical activity apps for other population groups suggest that commercial apps often lack evidence-based $\mathrm{BCT}$ s that have demonstrated efficacy for encouraging physical activity behavior change [15-17]. However, no such review of commercial apps designed to promote physical activity among pregnant women has been conducted. Thus, the appropriateness of these apps to promote physical activity during pregnancy is unknown. This review aimed to systematically evaluate the appropriateness of the apps designed to promote physical activity among pregnant women by using a systematic search and content analysis. Apps available through the Australian App Store and Google Play stores were accessed using the MARS tool for app quality and features. A taxonomy of BCT was also used to assess the presence of BCTs utilized within the apps, including BCTs that have demonstrated efficacy for promoting physical activity behavior change among pregnant women.

\section{Methods}

\section{Methodological Approach}

The methodological approach used in this study was informed by previous app reviews. These reviews explore app quality, features, and BCTs among apps designed to (1) improve diet, physical activity, and sedentary behavior in children and adolescents [15] and (2) provide nutritional advice to pregnant women [18].

\section{Search Strategy}

Systematic searches were conducted in the Australian App Store and Google Play stores between October 2018 and February 2019. Apps were identified using systematic combinations of the following search terms: pregnancy, pregnant, prenatal, postnatal, exercises, exercise, fitness, workout, and physical 
activity. These search term combinations were entered individually in the App Store and Google Play databases without any specified search categories, and search results were ordered by relevance (see Multimedia Appendix 1). As Google Play search results were capped at 250 apps, only the title and description of the first 250 relevant apps (in Google Play and App Store) were screened.

\section{Inclusion Criteria and Selection Process}

Apps were considered for inclusion if the description of the app in the stores specified pregnancy content and physical activity or exercise. Apps were included if they (1) targeted pregnant women, (2) had a focus on physical activity or exercise, (3) were available in English, and (4) had a user rating of at least 4.5 (scale range 1-5) in either of the stores (as similarly done elsewhere [17]) as a measure of app popularity. Both free and paid apps were eligible for inclusion; however, apps requiring external devices (eg, Kegel device, activity monitor, hardcopy books) were excluded due to limitations regarding device access and use. App selection and assessments were undertaken between October 2018 and April 2019.

As per best practice for systematic reviews [19], 2 reviewers (KLA and SC) independently reviewed the titles, images, and descriptions of each identified app for inclusion in the review. Disagreement was resolved by discussion and consensus with a third reviewer $(\mathrm{MH})$. Each of the eligible apps were examined independently by 2 of the 18 reviewers (ie, the authors), who were recognized as having expertise in behavior change or physical activity during pregnancy. If there was any notable disagreement in variance (eg, disagree versus agree) among the app assessment scores, a third reviewer would also be assigned. Each reviewer was allocated 4-6 apps to examine, determined by device accessibility (Apple or Android). Examination of apps included downloading, user testing, and assessing app features and quality criteria. Each app was allocated to an expert in behavior change and to an expert in physical activity during pregnancy for review. Incorporation of BCTs within each app were independently reviewed by 2 reviewers (KLA and SC). Any disagreements/discrepancies between reviewers KLA and $\mathrm{SC}$ were resolved by consultation with a third reviewer (MC). If an app was available in both App Store and Google Play, either version could be utilized for testing, regardless of differences in app user ratings. To maintain a consistent cost status and baseline assessment, if an app offered a free version and a paid version, the free version was included. To maintain this consistency, freemium content (ie, extra content at a cost) was not accessed and apps requiring paid subscriptions were excluded.

\section{Data Extraction}

Data extraction was conducted using a standard information spreadsheet and the Mobile App Rating Scale (MARS) [20]. Similar methods have been utilized in previous app reviews $[15,21]$. For all included apps, app name, developer, version, store (App Store, Google Play), cost (free, paid), average user rating (at least 4.5+), MARS focus points (what the app targets, eg, increase happiness/well-being, behavior change, entertainment, physical health), MARS theoretical background/strategies (eg, assessment, information/education, goal setting, advice/tips/strategies/skills training), and MARS technical aspects (eg, allows sharing, allows password protection, sends reminders) were extracted (see Multimedia Appendix 2 and Multimedia Appendix 3).

\section{App Features and Quality Assessment}

App features and quality were assessed using the MARS [20], as per prior app reviews [15,22]. The MARS consists of 19 items grouped in 4 domains: engagement (entertainment, interest, customization, interactivity, and target group); functionality (performance, ease of use, navigation, and gestural design); aesthetics (layout, graphics, and visual appeal); and information quality (accuracy of app description, goals, quality, and quantity of information, visual information, credibility, and evidence base). Additional MARS domains of subjective app quality (recommendation, potential use, payment, and overall rating) and likelihood of behavioral impact (awareness, knowledge, attitudes, intention to change, help seeking, and behavior change) were also included. Items were measured on a 5 -point scale ( $1=$ inadequate to $5=$ excellent) and a score for each domain was computed as the mean of the items in that domain; the overall score was computed as an average across the domains [20]. Final scores for each app and MARS items were calculated using the means of the reviewer scores (see Multimedia Appendix 4).

\section{BCT Identification}

The assessment of the presence or absence of BCTs for improving physical activity behavior was guided by the taxonomy of BCTs developed by Abraham and Michie [13]. A dichotomous score of 0 (absent) or 1 (present) was applied for each of the 26 BCTs, resulting in a total score of 0-26 (see Multimedia Appendix 5). This approach has been applied in similar app reviews and content analyses [15,23,24].

\section{Identification of Evidence-Based BCTs}

A brief literature search was employed to understand the BCTs that may be effective in supporting behavior change during pregnancy. A systematic review by Currie et al [12] described 6 BCTs that hold efficacy in reducing the decline of physical activity among pregnant women. These BCTs include prompt intention formation, prompt specific goal setting, prompt review of behavioral goals, prompt self-monitoring of behavior, provide feedback on performance, and provide opportunities for social comparison. As pregnancy-effective BCTs, these were specifically highlighted during analysis and results.

\section{Statistical Analyses}

In addition to descriptive statistics (mean, standard deviation, and range) calculated for each of the 6 MARS domains, frequencies (numbers and percentages) of each of the 26 BCTs included in the apps were calculated. Krippendorff's alpha $(\mathrm{K} \alpha)$ was used to evaluate interrater reliability for the app quality assessment and the presence of BCTs within the apps [25]. Spearman correlations were used to examine the relationships between app quality, number of technical app features, and number of BCTs incorporated in the apps. All statistical analyses were conducted using SPSS Statistics version 26.0 (IBM Corp) with significance levels set at $P<.05$. 


\section{Results}

\section{App Selection}

A flowchart of the app selection process is presented in Figure 1. A total of 7207 apps were identified and screened in the App Store and Google Play. Of these, 318 apps were further screened by description and 69 apps held content considered eligible for inclusion. The user rating criteria of $4.5+$ was applied and apps found to focus solely on postnatal physical activity/exercise were omitted. A total of 19 apps targeting physical activity during pregnancy were included in the content analysis and quality assessment.

Figure 1. PRISMA flow chart of the app selection process.

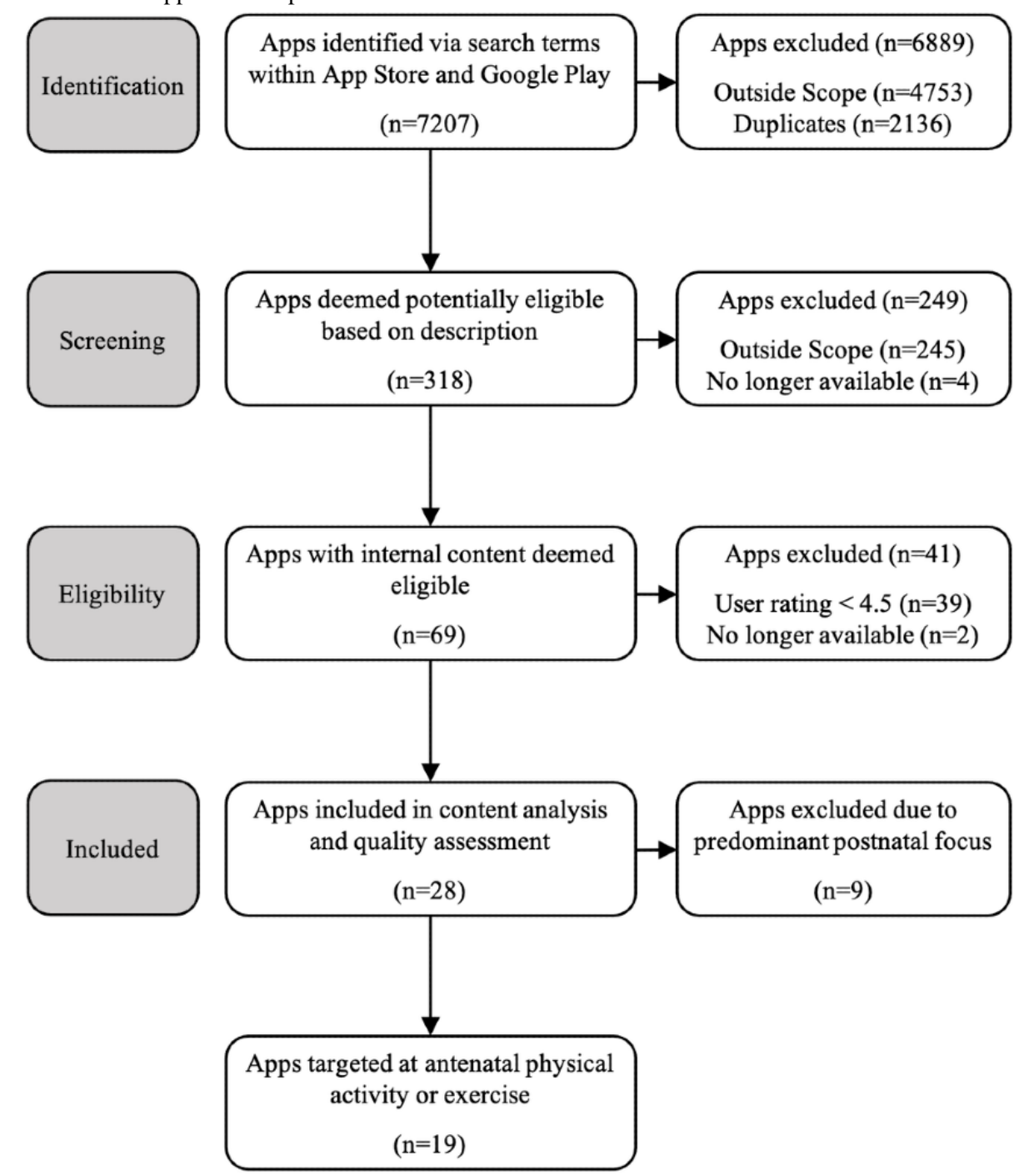

\section{App Characteristics}

Of the 19 reviewed antenatal physical activity apps, 13 were accessed via Google Play and 6 were accessed via App Store (see Multimedia Appendix 2). Apps were free to download, with the exception of one. The average star rating for the apps was 4.69 (SD 0.22), with a wide range of the number of users rating each app (mean 1875.16, SD 3549.82; range 1-13,000). On average, the 19 apps were found to contain few MARS-related categories: MARS focus points (mean 3.53 [SD 1.90]), MARS theoretical background/strategies (mean 3.58 [SD 1.98]), and MARS technical aspects (mean 1.74 [SD 1.73]). Figure 2 and Multimedia Appendix 3 detail the MARS categories for each of the 19 apps. 
Figure 2. Categories of focus points, theoretical background and strategies, and technical aspects of the Mobile App Rating Scale found in each app.

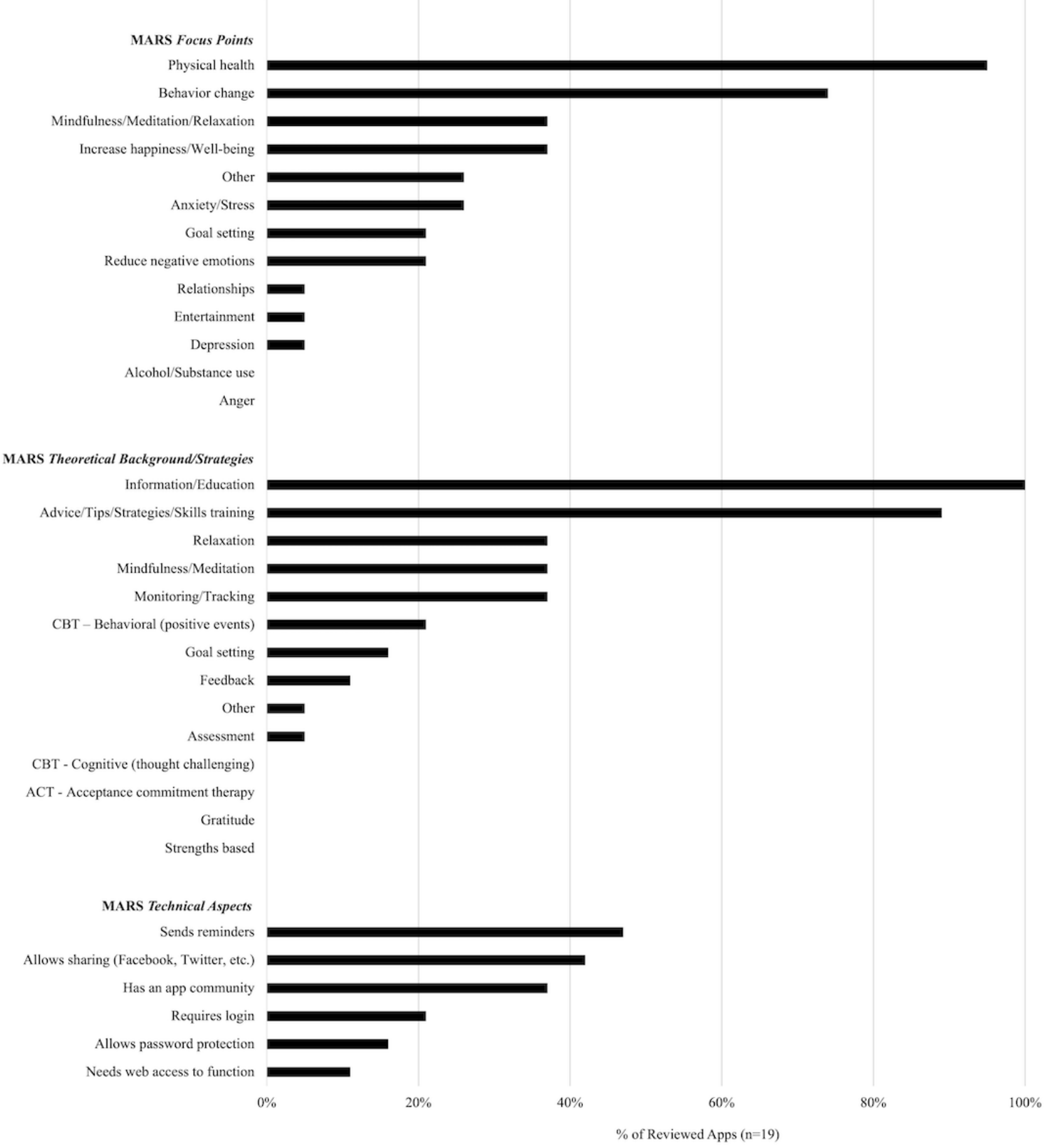

\section{App Quality}

The average MARS overall quality score was 3.5 out of 5 with a range of 2.4-4.3, which was considered to be of moderate quality. Functionality was the highest scoring domain (mean 4.2 [SD 0.5], followed by aesthetics (mean 3.7 [0.6]), information quality (mean 3.16 [SD 0.42]), and engagement (mean 3.01 [SD 0.9]). Subjective app quality (mean 2.5 [SD $0.6]$ ) and likelihood for behavioral impact (mean 2.5 [SD 0.6]) were equally the lowest scoring MARS domains. Table 1 provides a summary of the MARS scores. A detailed summary of the quality assessment of the included apps is presented in Multimedia Appendix 4. Interrater reliability [25] for app quality resulted in low reliability (mean $\mathrm{K} \alpha 0.3$, SD 0.37). However, there was no notable disagreement in variance (eg, disagree versus agree) among the app assessment scores; thus, a third reviewer was not required for any further app assessment. 
Table 1. Summary of the Mobile App Rating Scale (scale 1-5) scores across the 19 reviewed apps. ${ }^{\text {a }}$

\begin{tabular}{llll}
\hline Mobile App Rating Scale domain & Mean score (SD) & Median score (IQR) & Range of scores (min-max) \\
\hline Functionality & $4.22(0.49)$ & $4.3(0.75)$ & $1.7(3.3-5)$ \\
Aesthetics & $3.69(0.64)$ & $4(0.95)$ & $2.2(2.3-4.5)$ \\
Overall quality & $3.52(0.52)$ & $3.5(0.7)$ & $1.9(2.4-4.3)$ \\
Information quality & $3.19(0.42)$ & $3.2(0.75)$ & $1.4(2.6-4)$ \\
Engagement & $3.01(0.9)$ & $3(1.4)$ & $3.3(1.2-4.5)$ \\
Subjective quality & $2.54(0.64)$ & $2.5(0.95)$ & $2.3(1.5-3.8)$ \\
Likelihood of behavioral impact & $2.54(0.62)$ & $2.6(0.6)$ & $2.2(1.6-3.8)$ \\
\hline
\end{tabular}

${ }^{\mathrm{a}}$ Score $1=$ inadequate; score $5=$ excellent.

\section{Presence of BCTs}

The presence and types of BCTs found within the reviewed apps are presented in Figure 3 and Multimedia Appendix 5. Interrater reliability for evaluating the presence of BCTs in the apps was high (K $\alpha 0.85$, percent agreement $95 \%)$. All reviewed apps incorporated at least two BCTs. Commonly included BCTs included provide instructions $(18 / 19,95 \%)$, provide information on consequences $(17 / 19,89 \%)$, model or demonstrate the behavior (10/19, 53\%), and provide opportunities for social comparison $(8 / 19,42 \%)$. The average number of BCTs per app was 4.74 (SD 2.51) (range 2-10). Apps with the highest number of BCTs included were Pregnancy Week by Week Tracker (10 BCTs), iMum-Pregnancy \& Fertility (9 BCTs), and Pregnancy Tracker \& Countdown (9 BCTs).

Figure 3. Presence and types of behaviour change techniques found in the selected apps.

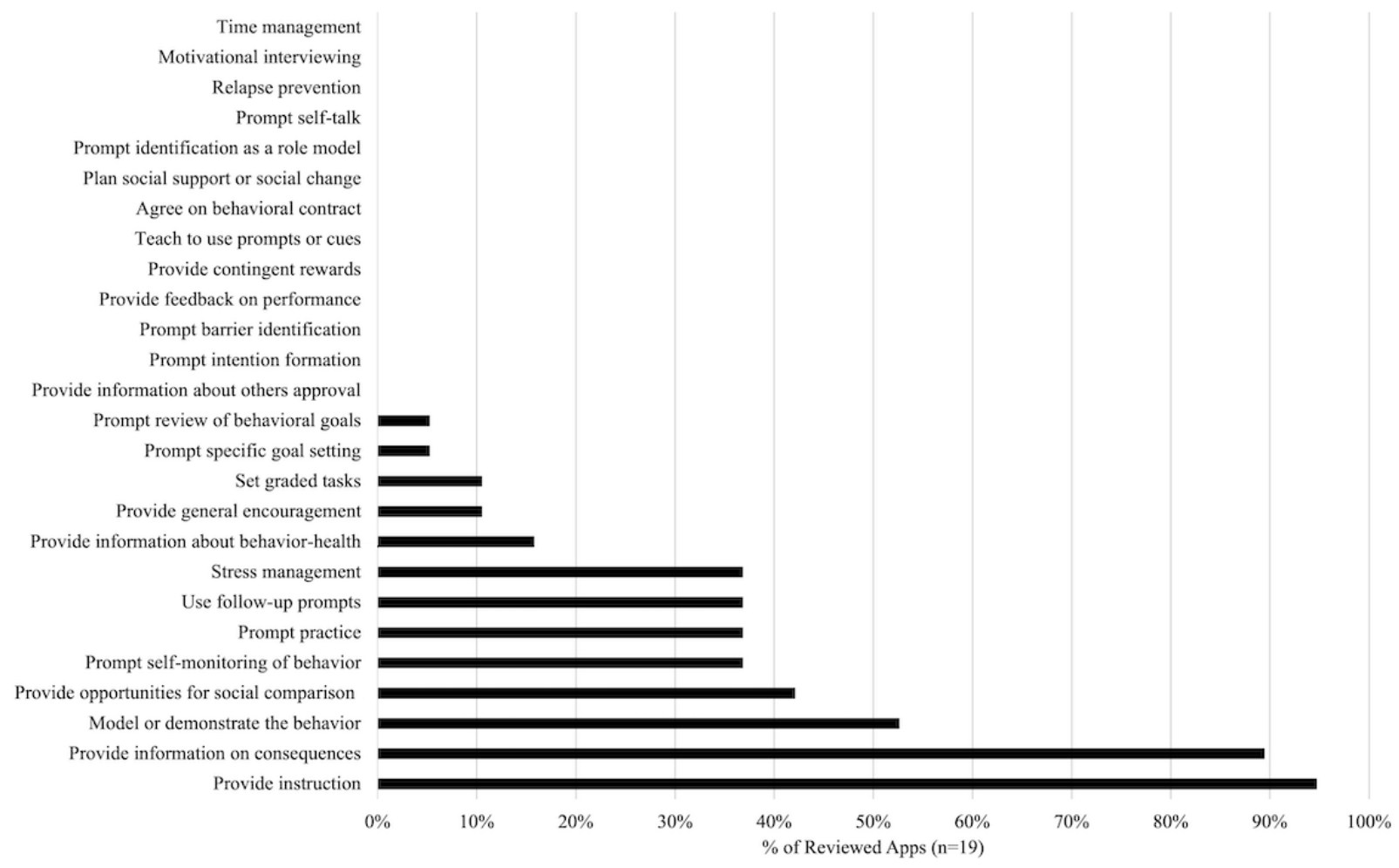

\section{Presence of Evidence-Based BCTs}

Currie et al [12] identified 6 common BCTs shown to have some efficacy in reducing the decline in physical activity behaviors among pregnant women, namely, prompt intention formation, prompt specific goal setting, prompt review of behavioral goals, prompt self-monitoring of behavior, provide feedback on performance, and provide opportunities for social comparison. Of the 19 apps reviewed in the present study, 11 apps contained at least one of these evidence-based BCTs (range 1-3) and 4 contained more than one of these evidence-based BCTs. Table 2 details the evidence-based BCTs included in each of the 11 apps. 
Table 2. Apps containing evidence-based techniques for behavior change during pregnancy. ${ }^{\mathrm{a}}$

\begin{tabular}{|c|c|c|c|c|c|c|}
\hline \multirow[t]{2}{*}{ App name } & \multicolumn{6}{|c|}{ Evidence-based behavior change techniques } \\
\hline & $\begin{array}{l}\text { Prompt intention } \\
\text { formation }(n=0)\end{array}$ & $\begin{array}{l}\text { Prompt } \\
\text { specific goal } \\
\text { setting }(n=1)\end{array}$ & $\begin{array}{l}\text { Prompt review of } \\
\text { behavioral goals } \\
(n=1)\end{array}$ & $\begin{array}{l}\text { Prompt self-monitor- } \\
\text { ing of behavior } \\
(\mathrm{n}=7)\end{array}$ & $\begin{array}{l}\text { Provide feedback on } \\
\text { performance }(\mathrm{n}=0)\end{array}$ & $\begin{array}{l}\text { Provide opportunities } \\
\text { for social comparison } \\
(\mathrm{n}=8)\end{array}$ \\
\hline Pregnancy+ $(\mathrm{n}=3)$ & & & $\checkmark$ & $\checkmark$ & & $\checkmark$ \\
\hline $\begin{array}{l}\text { Pregnancy Week by } \\
\text { Week Tracker }(n=3)\end{array}$ & & $\checkmark$ & & $\checkmark$ & & $\checkmark$ \\
\hline $\begin{array}{l}\text { Pregnancy Tracker } \\
\& \text { Countdown }(n=2)\end{array}$ & & & & $\checkmark$ & & $\checkmark$ \\
\hline $\begin{array}{l}\text { Pregnancy Work- } \\
\text { outs-Baby2Body } \\
(\mathrm{n}=2)\end{array}$ & & & & $\checkmark$ & & $\checkmark$ \\
\hline $\begin{array}{l}\text { 9Months Guide } \\
(\mathrm{n}=1)\end{array}$ & & & & & & $\checkmark$ \\
\hline Get Parenting & & & & & & $\checkmark$ \\
\hline $\begin{array}{l}\text { Pregnancy Tips. } \\
\text { Moms Pregnancy } \\
\text { App }(n=1)\end{array}$ & & & & & & \\
\hline $\begin{array}{l}\text { I'm Pregnant-Preg- } \\
\text { nancy Tracker }(n=1)\end{array}$ & & & & $\checkmark$ & & \\
\hline $\begin{array}{l}\text { iMum-Pregnancy \& } \\
\text { Fertility }(\mathrm{n}=1)\end{array}$ & & & & $\checkmark$ & & \\
\hline $\begin{array}{l}\text { Kegel Exercises } \\
(\mathrm{n}=1)\end{array}$ & & & & $\checkmark$ & & \\
\hline $\begin{array}{l}\text { Pregnancy Guide } \\
(\mathrm{n}=1)\end{array}$ & & & & & & $\checkmark$ \\
\hline $\begin{array}{l}\text { Pregnancy Health } \\
(\mathrm{n}=1)\end{array}$ & & & & & & $\checkmark$ \\
\hline
\end{tabular}

${ }^{\mathrm{a}}$ Apps not identified as containing a key behavior change technique are not noted in the table.

\section{Relationships Between App Quality, App Features, and BCTs}

Spearman correlations between the MARS overall quality, number of MARS focus points, number of MARS theoretical background/strategies, number of MARS technical aspects used in the app, MARS subjective quality, and the number of BCTs are presented in Table 3. The number of identified BCTs was positively associated with the MARS engagement score $(\rho=0.55$, $P=.01)$ and aesthetics score $(\rho=0.46, P=.046)$. MARS functionality, information quality, total MARS quality, and subjective quality were not significantly correlated with the number of BCTs. The number of app focus points was positively associated with the MARS engagement score $(\rho=0.58, P=.009)$, aesthetics score $(\rho=0.58, P=.008)$, total MARS quality score $(\rho=0.55, P=.02)$, and subjective quality score $(\rho=0.46, P=.048)$. The number of technical aspects within apps was positively correlated with the MARS engagement score $(\rho=0.63, P=.004)$ but not with any of the other MARS scores. Further, the MARS subjective quality scores were positively correlated with all other quality subscores (Table 3 ) and the total MARS quality score $(\rho=0.90, P<.001)$. 
Table 3. Correlations between Mobile App Rating Scale (MARS) overall quality, number of MARS focus points, number of MARS theoretical background/strategies, number of technical aspects, and MARS subjective quality.

\begin{tabular}{|c|c|c|c|c|c|}
\hline MARS subscales & $\begin{array}{l}\text { Behavior change } \\
\text { techniques }\end{array}$ & MARS focus points & $\begin{array}{l}\text { MARS theoretical } \\
\text { background/ } \\
\text { strategies }\end{array}$ & $\begin{array}{l}\text { MARS technical as- } \\
\text { pects }\end{array}$ & $\begin{array}{l}\text { MARS subjective } \\
\text { quality }\end{array}$ \\
\hline MARS engagement & $0.55^{\mathrm{a}}$ & $0.58^{\mathrm{b}}$ & $0.69^{\mathrm{b}}$ & $0.63^{\mathrm{b}}$ & $0.69^{\mathrm{b}}$ \\
\hline MARS functionality & 0.02 & 0.30 & 0.36 & -0.24 & $0.64^{\mathrm{b}}$ \\
\hline MARS aesthetics & $0.46^{\mathrm{a}}$ & $0.59^{\mathrm{b}}$ & $0.61^{\mathrm{b}}$ & 0.37 & $0.90^{\mathrm{b}}$ \\
\hline MARS information quality & 0.32 & 0.34 & 0.37 & 0.10 & $0.69^{\mathrm{b}}$ \\
\hline MARS overall quality & 0.45 & $0.55^{\mathrm{a}}$ & $0.67^{\mathrm{b}}$ & 0.31 & $0.90^{\mathrm{b}}$ \\
\hline MARS subjective quality & 0.42 & $0.46^{\mathrm{a}}$ & $0.60^{\mathrm{b}}$ & 0.36 & $-^{\mathrm{c}}$ \\
\hline
\end{tabular}

${ }^{\mathrm{a}}$ Correlation was significant at $P<.05$.

${ }^{\mathrm{b}}$ Correlation was significant at $P<.01$.

${ }^{\mathrm{c}}$ Not applicable.

\section{Discussion}

The primary aim of this app review was to assess app quality and features and presence of BCTs applied within commercial apps promoting physical activity in women during pregnancy. The secondary aim of this app review was to test the relationships between the 6 MARS domains of app quality and the number of MARS technical aspects, theoretical strategies, focus points, and BCTs. In summary, our findings demonstrate moderate MARS overall quality scores, with MARS domains of functionality and aesthetics scoring the highest and the domains of subjective app quality and likelihood for behavioral impact scoring the lowest. An average of 4.74 BCTs per app were used, with the most common BCTs being provide information on consequences and provide instructions. Most apps had none or one of the BCTs that demonstrated efficacy in promoting physical activity behaviors during pregnancy, with the most common BCTs being opportunities for social comparison and prompt self-monitoring of behavior.

Many commercially available physical activity apps for pregnant women were identified-with 69 relevant apps and 19 apps remaining after apps targeting postnatal physical activity $(n=9)$ and apps with user ratings lower than $4.5(n=39)$ were excluded. This finding demonstrates a market for apps targeting physical activity during pregnancy, which is not surprising, considering the findings of Lupton and Pedersen [9] who have previously reported that pregnant women frequently use pregnancy-related apps. The 69 apps identified as targeting exercise in pregnancy is comparable to the 51 apps Brown et al [18] identified as targeting nutrition in pregnancy. Pregnancy seems to be a time in women's lives in which they are actively seeking and using apps to support their behaviors, including physical activity.

The high MARS functionality scores and moderate-to-high MARS aesthetics scores may be a consequence of developers focusing on user experience for visually pleasing and user-friendly apps [26]. Although such features are important for attracting users, they do not translate to behavior change $[27,28]$. There is also room for improvement in app design in relation to the low MARS scores for quality of information and engagement. Given that pregnant women believe the information in apps has high credibility [9], it is important that apps ensure that evidence-based information is provided. It is also important that apps improve ratings of engagement, as engagement in app content is associated with behavior change [22].

Ratings of engagement could be improved by increasing the use of BCTs. Our study found an association between the number of BCTs and improved MARS engagement and aesthetics scores, which is consistent with the findings from a review investigating apps for weight management in adults [22]. The association between the number of BCTs and the engagement score could be due to the provision of additional content to engage in, increased usefulness, and perceived efficacy. It may be that BCTs are considered as enticements to potential users, thereby increasing engagement. If this is the case, this provides additional incentives for app developers to include BCTs. In line with that reported by Schoeppe et al [15] and Bardus et al [22], this study also found technical aspects of health behavior change apps to be associated with the MARS engagement score. Further, the number of theoretical strategies and the number of focus points were associated with MARS engagement, aesthetics, total quality, and subjective quality scores. Improving the number of technical aspects, theoretical strategies, and focus points may therefore help to further improve engagement of apps designed to promote physical activity among pregnant women.

An average of 4.74 BCTs were used in the reviewed apps. This is in line with the findings from previous research that found apps for physical activity promotion in adults to have an average of 4.2 [16] and 5.0 BCTs [24]. The number of BCTs identified in this review is, however, higher than those identified in a recent review exploring the number of BCTs among apps targeting nutrition behaviors in pregnancy [18], which reported an average of 3 BCTs per app [18]. We know that health behavior interventions using alternative modes of delivery (eg, websites) have improved outcomes when more BCTs are used [29]. Webb et al [29] suggest that this improvement may be a result of using a combination of BCTs that together target several stages and aspects of behavior change. However, we do 
not know the optimal number or combination of BCTs necessary to increase physical activity.

The most common BCTs identified in this study were provide information on consequences and provide instructions. Schoeppe et al [15] also found that providing instructions was a commonly used BCT in physical activity and diet apps for children and adolescents. This finding differs from the most common BCTs identified in apps targeting weight and physical activity in adults, which are goal setting, self-monitoring, and performance feedbacks [15,29]. Unfortunately, provide information on consequences and provide instructions have not been shown to be effective at improving physical activity behaviors in pregnant women [12] or even in the general adult population [27].

Most apps in this review had none or $1 \mathrm{BCT}$, which previously demonstrated efficacy in behavior change during pregnancy. Including more of these evidence-based BCTs in the context of pregnancy may improve the ability of the apps to support pregnant women in increasing their physical activity [12]. The evidence-based BCTs of intention formation, goal setting, review of goals, and feedback on performance have been successfully implemented within physical activity apps targeting other population groups $[15,16]$. This demonstrates that it is feasible to deliver these evidence-based BCTs through an app. In particular, feedback on performance was identified as one of the most commonly used BCTs in apps targeting physical activity, diet, and sedentary behavior in children and adults [15]. Feedback on performance requires the measurement of behavior (eg, frequency, intensity of exercise), and apps that incorporate wearable devices (eg, Fitbit, Garmin) to measure physical activity behavior may be more likely to provide feedback on performance [30]. Therefore, the exclusion of apps that required the use of a wearable device may partially explain the finding of low use of feedback on performance in this study. The most common technical features were sends reminders $(9 / 19,47 \%)$ and allows sharing $(8 / 19,42 \%)$. There is evidence to suggest that reminders improve the effectiveness of health behavior change interventions [31]; however, other evidence suggests that reminders can hinder habit formation, which may have an impact on long-term behavior change [32]. The high use of sharing is contradictory to the findings that adults find social media features unnecessary and off-putting in health behavior change apps [33]. The removal of sharing features may improve engagement ratings.

The strengths of this study include the systematic search for apps from both App Store and Google Play, the use of an established taxonomy for identifying BCTs, the use of the
MARS instrument to assess the quality of apps, and the inclusion of apps rated above $4.5 / 5$. Further, app ratings were performed by a minimum of two reviewers following best practices for conducting systematic reviews [19]. Good interrater reliability was found for the scoring of BCTs.

The limitations of this study include the exclusion of apps that required use of a wearable device and low interrater reliability for the scoring of app quality through the MARS scale. This may be due to the subjective nature of some sections of the scale. What one reviewer may find aesthetically pleasing, functional, or engaging, another reviewer may not. To account for these differences, the average of the 2 scores was used as the final score for each domain. The temporal relevancy of the results from this study may also be considered a limitation, despite the search being conducted less than 12 months ago because unlike traditional literature where the content remains consistent and unchanged once published, apps are extremely fluid, resulting in frequent updates and modifications to their contents and features. Despite these limitations, this study is the first of its kind and provides valuable real-world findings and implications that are highly relevant to this field as well as the greater audience. Future research should test the overall effectiveness of commercial apps designed to promote physical activity among pregnant women. Finally, research examining the accuracy of app content and the expertise of developers should also be of high priority.

In conclusion, the use of apps for physical activity advice and support in pregnancy is rising. Therefore, an understanding of their quality and inclusion of effective BCTs is required. This is the first study to investigate the quality of popular commercially available apps for promoting physical activity in women during pregnancy. The findings of moderate app quality, with the highest ratings for functionality and aesthetics, indicate that the apps are user-friendly. However, the low use of evidence-based BCTs for changing physical activity behavior in women during pregnancy indicates that the popular commercial apps currently available may not be effective at promoting physical activity behavior in the pregnant population. More effort needs to be placed on incorporating components most likely to influence behavior change, which is ultimately what they are developed for, while maintaining good functionality. Developers should continue to provide self-monitoring and social comparison and ensure that these components are engaging and effective. In addition, intention formation, goal setting, review of goals, and feedback on performance should be incorporated in new apps and new versions of existing apps.

\section{Acknowledgments}

The authors received no specific funding for this work. Other funding support is as follows: SS is supported by an Early Career Fellowship (GNT1125586) from the National Health and Medical Research Council of Australia and by a Postdoctoral Fellowship (ID 101240) from the National Heart Foundation of Australia. SA is funded by a National Heart Foundation Postdoctoral Fellowship (ID 102609). AA and NC are supported by the National Institutes of Health, which fund the Nutrition Obesity Research Center (P30DK072476) and the National Institute of Nursing Research (R01 NR017644). GPM is supported by a University of Queensland International Postgraduate Research Scholarship. TSN is funded by a Mitacs Fellowship supported by The Society of Obstetricians and Gynecologists of Canada. 
LM is funded by a National Heart Foundation Future Leader Fellowship and also funded by the Australian Government's Medical Research Future Fund (MRFF; TABP-18-0001). The MRFF provides funding to support health and medical research and innovation, with the objective of improving the health and well-being of Australians. MRFF funding has been provided to The Australian Prevention Partnership Centre under the MRFF Boosting Preventive Health Research Program. Further information on the MRFF is available in the Australian Government Department of Health website.

\section{Authors' Contributions}

MH conceptualized and designed the study, with input from KLA and SS. KLA, SC, and MH screened the apps for eligibility based on app descriptions. All authors downloaded and tested the apps, extracted the data, and contributed to content analysis and quality assessment. KLA and SC identified BCTs and sought confirmation from other reviewers. KLA analyzed the data. KLA, MH, LV, and SA drafted the manuscript. All authors were involved in the interpretation of data and critical revision of the manuscript, and they read and approved the final manuscript.

\section{Conflicts of Interest}

None declared.

\section{Multimedia Appendix 1}

Search strategy and search results. [DOC File, 78 KB-Multimedia Appendix 1]

\section{Multimedia Appendix 2}

App characteristics.

[DOC File, 63 KB-Multimedia Appendix 2]

\section{Multimedia Appendix 3}

Mobile App Rating Scale categories.

[DOC File, 63 KB-Multimedia Appendix 3]

\section{Multimedia Appendix 4}

Mean Mobile App Rating Scale scores and interrater reliability.

[DOC File, 65 KB-Multimedia Appendix 4]

\section{Multimedia Appendix 5}

Behavior change techniques and interrater reliability.

[DOC File, 107 KB-Multimedia Appendix 5]

\section{References}

1. Artal R. Exercise during pregnancy and the postpartum period. UpToDate. 2017 Apr. URL: https://www.uptodate.com/ contents/exercise-during-pregnancy-and-the-postpartum-period\# [accessed 2019-01-01]

2. Harrison CL, Brown WJ, Hayman M, Moran LJ, Redman LM. The Role of Physical Activity in Preconception, Pregnancy and Postpartum Health. Semin Reprod Med 2016 Mar;34(2):e28-e37 [FREE Full text] [doi: 10.1055/s-0036-1583530] [Medline: 27169984]

3. da Silva SG, Ricardo LI, Evenson KR, Hallal PC. Leisure-Time Physical Activity in Pregnancy and Maternal-Child Health: A Systematic Review and Meta-Analysis of Randomized Controlled Trials and Cohort Studies. Sports Med 2017 Feb;47(2):295-317. [doi: 10.1007/s40279-016-0565-2] [Medline: 27282925]

4. Hayman M, Short C, Reaburn P. An investigation into the exercise behaviours of regionally based Australian pregnant women. J Sci Med Sport 2016 Aug;19(8):664-668. [doi: 10.1016/j.jsams.2015.09.004] [Medline: 26481261]

5. Coll CV, Domingues MR, Gonçalves H, Bertoldi AD. Perceived barriers to leisure-time physical activity during pregnancy: A literature review of quantitative and qualitative evidence. J Sci Med Sport 2017 Jan;20(1):17-25. [doi: 10.1016/j.jsams.2016.06.007] [Medline: 27372276]

6. Tripp N, Hainey K, Liu A, Poulton A, Peek M, Kim J, et al. An emerging model of maternity care: smartphone, midwife, doctor? Women Birth 2014 Mar;27(1):64-67. [doi: 10.1016/j.wombi.2013.11.001] [Medline: 24295598]

7. Lee Y, Moon M. Utilization and Content Evaluation of Mobile Applications for Pregnancy, Birth, and Child Care. Healthc Inform Res 2016 Apr;22(2):73-80 [FREE Full text] [doi: 10.4258/hir.2016.22.2.73] [Medline: 27200216] 
8. van den Heuvel JF, Groenhof TK, Veerbeek JH, van Solinge WW, Lely AT, Franx A, et al. eHealth as the Next-Generation Perinatal Care: An Overview of the Literature. J Med Internet Res 2018 Jun 05;20(6):e202 [FREE Full text] [doi: 10.2196/jmir.9262] [Medline: 29871855]

9. Lupton D, Pedersen S. An Australian survey of women's use of pregnancy and parenting apps. Women Birth 2016 Aug;29(4):368-375. [doi: 10.1016/j.wombi.2016.01.008] [Medline: 26874938]

10. Samdal GB, Eide GE, Barth T, Williams G, Meland E. Effective behaviour change techniques for physical activity and healthy eating in overweight and obese adults; systematic review and meta-regression analyses. Int J Behav Nutr Phys Act 2017 Mar 28;14(1):42 [FREE Full text] [doi: 10.1186/s12966-017-0494-y] [Medline: 28351367]

11. Michie S, Carey RN, Johnston M, Rothman AJ, de Bruin M, Kelly MP, et al. From Theory-Inspired to Theory-Based Interventions: A Protocol for Developing and Testing a Methodology for Linking Behaviour Change Techniques to Theoretical Mechanisms of Action. Ann Behav Med 2018 May 18;52(6):501-512 [FREE Full text] [doi: 10.1007/s12160-016-9816-6] [Medline: 27401001]

12. Currie S, Sinclair M, Murphy MH, Madden E, Dunwoody L, Liddle D. Reducing the decline in physical activity during pregnancy: a systematic review of behaviour change interventions. PLoS One 2013;8(6):e66385 [FREE Full text] [doi: 10.1371/journal.pone.0066385] [Medline: 23799096]

13. Abraham C, Michie S. A taxonomy of behavior change techniques used in interventions. Health Psychol 2008 May;27(3):379-387. [doi: 10.1037/0278-6133.27.3.379] [Medline: 18624603]

14. Zinsser LA, Stoll K, Wieber F, Pehlke-Milde J, Gross MM. Changing behaviour in pregnant women: A scoping review. Midwifery 2020 Jun;85:102680 [FREE Full text] [doi: 10.1016/j.midw.2020.102680] [Medline: 32151875]

15. Schoeppe S, Alley S, Rebar AL, Hayman M, Bray NA, Van Lippevelde W, et al. Apps to improve diet, physical activity and sedentary behaviour in children and adolescents: a review of quality, features and behaviour change techniques. Int $\mathrm{J}$ Behav Nutr Phys Act 2017 Jun 24;14(1):83 [FREE Full text] [doi: 10.1186/s12966-017-0538-3] [Medline: 28646889]

16. Conroy DE, Yang C, Maher JP. Behavior change techniques in top-ranked mobile apps for physical activity. Am J Prev Med 2014 Jun;46(6):649-652. [doi: 10.1016/j.amepre.2014.01.010] [Medline: 24842742]

17. Milne-Ives M, Lam C, De Cock C, Van Velthoven MH, Meinert E. Mobile Apps for Health Behavior Change in Physical Activity, Diet, Drug and Alcohol Use, and Mental Health: Systematic Review. JMIR Mhealth Uhealth 2020 Mar 18;8(3):e17046 [FREE Full text] [doi: 10.2196/17046] [Medline: 32186518]

18. Brown HM, Bucher T, Collins CE, Rollo ME. A review of pregnancy iPhone apps assessing their quality, inclusion of behaviour change techniques, and nutrition information. Matern Child Nutr 2019 Jul;15(3):e12768 [FREE Full text] [doi:

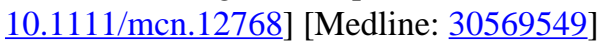

19. Moher D, Liberati A, Tetzlaff J, Altman DG, PRISMA Group. Preferred reporting items for systematic reviews and meta-analyses: the PRISMA statement. PLoS Med 2009 Jul 21;6(7):e1000097 [FREE Full text] [doi: 10.1371/journal.pmed.1000097] [Medline: 19621072]

20. Stoyanov SR, Hides L, Kavanagh DJ, Zelenko O, Tjondronegoro D, Mani M. Mobile app rating scale: a new tool for assessing the quality of health mobile apps. JMIR Mhealth Uhealth 2015 Mar 11;3(1):e27 [FREE Full text] [doi: 10.2196/mhealth.3422] [Medline: 25760773]

21. Schoffman DE, Turner-McGrievy G, Jones SJ, Wilcox S. Mobile apps for pediatric obesity prevention and treatment, healthy eating, and physical activity promotion: just fun and games? Transl Behav Med 2013 Sep;3(3):320-325 [FREE Full text] [doi: 10.1007/s13142-013-0206-3] [Medline: 24073184]

22. Bardus M, van Beurden SB, Smith JR, Abraham C. A review and content analysis of engagement, functionality, aesthetics, information quality, and change techniques in the most popular commercial apps for weight management. Int J Behav Nutr Phys Act 2016 Mar 10;13:35 [FREE Full text] [doi: 10.1186/s12966-016-0359-9] [Medline: 26964880]

23. Direito A, Dale LP, Shields E, Dobson R, Whittaker R, Maddison R. Do physical activity and dietary smartphone applications incorporate evidence-based behaviour change techniques? BMC Public Health 2014 Jun 25;14:646 [FREE Full text] [doi: 10.1186/1471-2458-14-646] [Medline: 24965805]

24. Middelweerd A, Mollee JS, van der Wal CN, Brug J, Te Velde SJ. Apps to promote physical activity among adults: a review and content analysis. Int J Behav Nutr Phys Act 2014 Jul 25;11:97 [FREE Full text] [doi: 10.1186/s12966-014-0097-9] [Medline: 25059981]

25. Hayes AF, Krippendorff K. Answering the Call for a Standard Reliability Measure for Coding Data. Communication Methods and Measures 2007 Apr;1(1):77-89. [doi: 10.1080/19312450709336664]

26. Schnall R, Rojas M, Bakken S, Brown W, Carballo-Dieguez A, Carry M, et al. A user-centered model for designing consumer mobile health (mHealth) applications (apps). J Biomed Inform 2016 Apr;60:243-251 [FREE Full text] [doi: 10.1016/j.jbi.2016.02.002] [Medline: 26903153]

27. Michie S, Abraham C, Whittington C, McAteer J, Gupta S. Effective techniques in healthy eating and physical activity interventions: a meta-regression. Health Psychol 2009 Nov;28(6):690-701. [doi: 10.1037/a0016136] [Medline: 19916637]

28. Schoeppe S, Alley S, Van Lippevelde W, Bray NA, Williams SL, Duncan MJ, et al. Efficacy of interventions that use apps to improve diet, physical activity and sedentary behaviour: a systematic review. Int J Behav Nutr Phys Act 2016 Dec 07;13(1):127 [FREE Full text] [doi: 10.1186/s12966-016-0454-y] [Medline: 27927218] 
29. Webb TL, Joseph J, Yardley L, Michie S. Using the internet to promote health behavior change: a systematic review and meta-analysis of the impact of theoretical basis, use of behavior change techniques, and mode of delivery on efficacy. $\mathrm{J}$ Med Internet Res 2010 Feb 17;12(1):e4 [FREE Full text] [doi: 10.2196/jmir.1376] [Medline: 20164043]

30. Lyons EJ, Lewis ZH, Mayrsohn BG, Rowland JL. Behavior change techniques implemented in electronic lifestyle activity monitors: a systematic content analysis. J Med Internet Res 2014 Aug 15;16(8):e192 [FREE Full text] [doi: 10.2196/jmir.3469] [Medline: 25131661]

31. Fry JP, Neff RA. Periodic prompts and reminders in health promotion and health behavior interventions: systematic review. J Med Internet Res 2009 May 14;11(2):e16 [FREE Full text] [doi: 10.2196/jmir.1138] [Medline: 19632970]

32. Stawarz K, Cox A, Blandford A. Beyond Self-Tracking and Reminders: Designing Smartphone Apps That Support Habit Formation. 2015 Presented at: 33rd Annual AMC Conference on Human Factors in Computing Systems; April, 2015; Seoul, Republic of Korea. [doi: 10.1145/2702123.2702230]

33. Dennison L, Morrison L, Conway G, Yardley L. Opportunities and challenges for smartphone applications in supporting health behavior change: qualitative study. J Med Internet Res 2013 Apr 18;15(4):e86 [FREE Full text] [doi: 10.2196/jmir.2583] [Medline: 23598614]

\title{
Abbreviations \\ BCT: behavior change technique \\ K $\alpha$ : Krippendorff's alpha \\ MARS: Mobile App Rating Scale
}

\author{
Edited by G Eysenbach; submitted 18.08.20; peer-reviewed by B Wollesen, P Grace-Farfaglia; comments to author 01.10.20; revised \\ version received 21.10.20; accepted 17.01.21; published 07.04.21 \\ Please cite as: \\ Hayman M, Alfrey KL, Cannon S, Alley S, Rebar AL, Williams S, Short CE, Altazan A, Comardelle N, Currie S, Denton C, Harrison \\ CL, Lamerton T, Mena GP, Moran L, Mottola M, Nagpal TS, Vincze L, Schoeppe S \\ Quality, Features, and Presence of Behavior Change Techniques in Mobile Apps Designed to Improve Physical Activity in Pregnant \\ Women: Systematic Search and Content Analysis \\ JMIR Mhealth Uhealth 2021;9(4):e23649 \\ URL: https://mhealth.jmir.org/2021/4/e23649 \\ doi: $10.2196 / 23649$ \\ PMID:
}

CMelanie Hayman, Kristie-Lee Alfrey, Summer Cannon, Stephanie Alley, Amanda L Rebar, Susan Williams, Camille E Short, Abby Altazan, Natalie Comardelle, Sinead Currie, Caitlin Denton, Cheryce L Harrison, Tayla Lamerton, Gabriela P Mena, Lisa Moran, Michelle Mottola, Taniya S Nagpal, Lisa Vincze, Stephanie Schoeppe. Originally published in JMIR mHealth and uHealth (http://mhealth.jmir.org), 07.04.2021. This is an open-access article distributed under the terms of the Creative Commons Attribution License (https://creativecommons.org/licenses/by/4.0/), which permits unrestricted use, distribution, and reproduction in any medium, provided the original work, first published in JMIR mHealth and uHealth, is properly cited. The complete bibliographic information, a link to the original publication on http://mhealth.jmir.org/, as well as this copyright and license information must be included. 